\title{
Assessment of Physiological Basis of Yield Variation in Small Millets under Rainfed Condition
}

\author{
R. Samundeswari ${ }^{1 *}$, D. Durga Devi ${ }^{2}$, P. Jayakumar ${ }^{2}$ and N. Jeyapandiyan ${ }^{2}$ \\ ${ }^{I}$ Department of Crop Physiology, ${ }^{2}$ Agro Climate Research Centre \\ Tamil Nadu Agricultural University, Coimbatore - 641003 \\ Tamil Nadu India \\ *Corresponding author
}

\section{Keywords}

Small millets,

Physiological traits,

Photosynthetic rate,

Stomatal

Article Info

Accepted:

17 June 2018

Available Online:

10 July 2018

\section{A B S T R A C T}

Physiological traits play an important role in crop growth and development. Comparative investigation on small millets, with respect different physiological traits such as leaf area index, leaf area duration, specific leaf weight, chlorophyll content, gas exchange parameters etc. and their relationship with grain yields were meager, and will be useful in small millets improvement. Therefore, this study aims to investigate the physiological traits and their relationship with grain yields five small millets (foxtail millet, proso millet, kodo millet, little millet and barnyard millet). Physiological traits such as leaf number, leaf area, specific leaf weight, chlorophylls a, b, and total chlorophyll, SPAD reading, photosynthetic rate and transpiration rate have reached their maximum value at grain development stage in all the crops, while leaf area index was the maximum at grain development stage, highest root length was achieved at maturity stage, and highest stomatal conductance was at flowering stage. Among different cultivars within the each crop, a cultivar having high leaf number, leaf area, leaf area index, leaf area duration, specific leaf weight, chlorophyll a, b, and total chlorophyll, chlorophyll fluorescence ratio, SPAD reading, photosynthetic rate, transpiration rate, stomatal conductance and root length, had produced higher grain yield. This shows importance of these traits in for enhanced yields in small millets.

\section{Introduction}

Small millets such as finger millet, foxtail millet, proso millet, kodo millet, little millet and barnyard millets are considered as important nutri-rich and climate-smart crops and are adapted to diverse environments (Vetriventhan et al., 2015 and Upadhyaya et al., 2008). Small millets play an important role in diversifying agriculture, supporting traditional farming systems and improving food and nutritional security particularly in marginal lands. Small millets are grown in India, China, Russia, Japan., USA and other African and East Asian countries. In India, the cultivation of small millets are cultivated in limited area of $2.32 \mathrm{~m}$ ha and occupy about 9.7 lakhs ha with a production of 4.67 lakhs tons, with a productivity of $480 \mathrm{~kg} / \mathrm{ha}$ (averaged between 2006-2010). 
Potential yields of up to 3 tons in small millets were reported (http://www.aicrpsm. res.in/Reports.html), indicating a large yield gaps, and great opportunity to enhance productivity following improved crop management practices and cultivation high yielding cultivars. Comparative investigation on small millets, with respect different physiological traits such as leaf area index, leaf area duration, specific leaf weight, chlorophyll content, etc. and their relationship with grain yields were meager, and will be useful in small millets improvement. Therefore, this study aims to investigate the physiological traits and their relationship with grain yields.

\section{Materials and Methods}

The experiment was conducted at Tamil Nadu Agricultural University, Coimbatore situated at $11 \mathrm{~N}^{\circ}$ and $77 \mathrm{E}^{\circ}$ longitude with at an altitude of $426.7 \mathrm{~m}$ above mean sea level. This study included two cultivars each of barnyard millet (Co 1 and Co 2) and kodo millet (Co 3, APK), three cultivars each of proso millet (Co $3, \mathrm{Co}$ 4 and Co 5), little millet (Co 2, Co 3 and Co 4) and foxtail millet (Co 5, Co 6 and Co 7). Together, 13 cultivars of five small millets were planted in randomized complete block design with three replications. The experiment received NPK in the form of urea, single super phosphate and muriate of potash, respectively at the rate of 44: 22: $15 \mathrm{~kg} / \mathrm{ha}$. Full dose of $\mathrm{P}$ was applied as basal, whereas, $\mathrm{N}$ was applied in two splits, one as basal and another at 30 days after sowing (DAS). Potassium in the form of Muriate of potash was applied at $20^{\text {th }}$ and $40^{\text {th }}$ DAS. The observations on physiological traits and Gas exchange parameters were recorded at seedling (20-25 DAS), vegetative (30-35 DAS), flowering (4055 DAS), grain development (60-70 DAS) and grain maturation (75-85 DAS) stages of the crop. All the observations were made from ten randomly selected plants from each replication of all the treatments. The physiological traits such as number of leaves, leaf area, leaf area index (LAI), leaf area duration (LAD), specific leaf weight (SLW), root length, Chlorophyll a, b and total chlorophyll content were estimated. The number of leaves per plant was determined by counting the leaves from the base to the tip of the plant. Leaf area for the whole sampling unit was measured by using Leaf Area Meter (Licor Model 3100) and expressed as $\mathrm{cm}^{2}$ plant $^{-1}$. The Leaf Area Index (LAI) was calculated by employing the formula of Williams (1946).

\section{$\mathrm{LAI}=$}

leaf area per plant/ground area occupied by the plant

Leaf Area Duration $(\mathrm{LAD}=$

$\frac{\mathrm{L} 1+\mathrm{L} 2}{2}(\mathrm{t} 2-\mathrm{t} 1)$

where, $\mathrm{L}_{1}=\mathrm{LAI}$ at first stage, $\mathrm{L}_{2}=\mathrm{LAI}$ at second stage, $\mathrm{t} 1-\mathrm{t} 2=$ Time interval in days) was determined using the formula of Power et al. (1967) and expressed in days. Specific leaf weight (SLW= leaf dry weight/leaf area) was determined by using the formula given by Pearce et al. (1968) and expressed as $\mathrm{mg} \mathrm{cm}^{-2}$.

The plant was uprooted with minimum damage to the roots and the root length from the cotyledonary node to the root tip was measured and expressed as $\mathrm{cm}$. Chlorophyll a, $\mathrm{b}$ and total chlorophyll content, were estimated in a fully expanded young leaf as per the method of Arnon (1949) and expressed as $\mathrm{mg} \mathrm{g}^{-1}$ fresh weight. Chlorophyll index in leaves was measured using SPAD meter. Photosynthetic rate, transpiration rate and stomatal conductance was measured following portable Photosynthesis System (PPS) (Model LI-6400 of LICOR Inc., Lincoln, Nebraska, USA) equipped with a halogen lamp (640002B LED) positioned on the cuvette. Totally, 
three measurements were taken in the same leaf. The fully expanded young leaf was inserted in a $3 \mathrm{~cm}^{2}$ leaf chamber and PPFD at $1200 \mu \mathrm{mol}$ photons $\mathrm{m}^{-2} \mathrm{~s}^{-1}$ and relative humidity $(50-55 \%)$ were set. The readings were taken between 11.00 am to $12.30 \mathrm{pm}$. Using PPS, the following gas exchange parameters were recorded and the values expressed as in parentheses.

Transpiration rate $\left(\mathrm{mmol} \mathrm{H}_{2} \mathrm{O} \mathrm{m}^{-2} \mathrm{~s}^{-1}\right)$

Stomatal conductance $\left(\mathrm{mmol} \mathrm{H}_{2} \mathrm{O} \mathrm{m}^{-2} \mathrm{~s}^{-1}\right.$ )

Photosynthetic rate $\left(\mu \mathrm{mol} \mathrm{CO} \mathrm{Cm}^{-2} \mathrm{~s}^{-1}\right)$

The data collected on the different parameters were statistically analyzed by the ' $F$ ' test for significance as suggested by Gomez and Gomez (2010). The critical difference (CD) was computed at $5 \%$ probability. Biochemical traits at different crop growth stages were compared following Newman and Keul's test (Newman 1939; Keuls 1952) using the GenStat 17th edition (http://www.genstat. co.uk).

\section{Results and Discussion}

\section{Physiological traits}

Physiological traits such as leaf number, leaf area, leaf area index, leaf area duration, specific leaf weight, chlorophyll a, chlorophyll $\mathrm{b}$ and total chlorophyll content and SPAD reading (Soil Plant Analysis Development) were recorded at five different growth stages of small millets (Table 1). Leaf number of was the highest in little millet at grain development (13.20), grain maturation (11.93) and harvest (6.90) stages while barnyard millet had the maximum number of leaf at flowering (7.60) and grain development (8.20) stages. Among five crop growth stages, the maximum leaf was reached at grain development stage (5.60 in kodo millet to 13.20 in little millet) in all small millets investigated (Table 2). Leaf area, leaf area index and leaf area duration ware maximum in barnyard millet in four out of five growth stages compared to other crops investigated (Table 1), and reached the highest leaf area during grain development stage, leaf area index at grain maturation stage and leaf area duration in either of grain development or grain maturation stages. Specific leaf weight was the highest in foxtail millet at all five growth stages, and it has reached the maximum at grain developmental stage in all the crops. Chlorophyll a, b and total chlorophyll contents reached the maximum at grain development stage in all the crops.

Chlorophyll $\mathrm{b}$ was the maximum in finger millet in first four stages while kodo millet had the highest chlorophyll $b$ content at maturity. Chlorophyll a was the maximum at vegetative stage in barnyard millet, flowering stage in kodo millet, grain development and maturation stages in proso millet and at harvest in little millet. Total chlorophyll content was the maximum in kodo millet in all crop growth stages except at grain maturation stage and reached the maximum at flowering stage in all five crops. Foxtail millet had slightly higher chlorophyll fluorescence ( $\mathrm{Fv} / \mathrm{Fm}$ ratio) and in all crops, it is maximum at grain development stage and low at maturity stage. The SPAD reading was maximum in foxtail millet in vegetative to grain maturation stage while barnyard millet had the maximum SPAD value at harvest.

\section{Gas exchange parameters}

Gas exchange parameters such as photosynthetic rate, transpiration rate and stomatal conductance were recorded at vegetative, flowering, panicle initiation and maturation stages in 13 cultivars of five small millets. Photosynthetic rate was the maximum in proso millet at vegetative, flowering and grain maturity stage compared to other crops, 
and it reached highest at grain development stage and significantly differed with other stages in all the crops. Transpiration rate was the maximum at vegetative stage and grain development stage in proso millet, flowering stage in barnyard millet and maturity stage in little millet, and it reached the maximum at grain development stage in all the crops and differed significantly. Stomatal conductance was maximum in proso millet at vegetative, grain development and maturity stages and was reached the highest at flowering stage in all five small millets studied.

Relationship of physiological traits with grain yield

Two cultivars each in barnyard and kodo millets, and three cultivars each in foxtail millet, proso, and little millets were used in this study. Flowering duration of these cultivars varied from 40 to 65 DAS. Except kodo millet, remaining four crops' cultivars flowered within 52 DAS, and matured in less than 95 DAS. Grain yields of small millets cultivars varied from $1133 \mathrm{~kg} / \mathrm{ha}$ (APK of kodo millet) to $3499 \mathrm{~kg} \mathrm{ha}^{-1}$ (Co 7 of foxtail millet), and straw yield varied from $5083 \mathrm{~kg}$ $\mathrm{ha}^{-1}$ to $7666 \mathrm{~kg} / \mathrm{ha}$. Harvest index varied from 0.27 to 4.10 among cultivars. Harvest index was highest in kodo millet ( 0.39 to 0.41 , mean $0.40)$ and was lowest in foxtail millet (0.27 to 0.32 , mean of 0.28). The foxtail millet cultivars yielded an average of $3033 \mathrm{~kg} / \mathrm{ha}$ followed by proso millet $(2877 \mathrm{~kg} / \mathrm{ha})$, and least was in kodo millet $(1575 \mathrm{~kg} / \mathrm{ha})$. Within each crop, a cultivar having high leaf number, leaf area, leaf area index, leaf area duration, specific leaf weight, chlorophyll a, b, and total chlorophyll, chlorophyll florescence ratio, SPAD reading and root length had produced higher grain yield (Table 3 to 7 ).

Table.1 Gas exchange parameters of small millets at different growth stages

\begin{tabular}{|l|c|c|c|c|}
\hline \multirow{2}{*}{ Crop } & \multicolumn{3}{|c|}{ Growth stage } \\
\cline { 2 - 5 } & $\begin{array}{c}\text { Vegetative } \\
\text { state }\end{array}$ & Flowering & $\begin{array}{c}\text { Grain } \\
\text { development }\end{array}$ & $\begin{array}{c}\text { Grain } \\
\text { maturity }\end{array}$ \\
\hline Photosynthetic rate & & & & \\
\hline Barnyard millet & $29.90 \mathrm{a}$ & $35.45 \mathrm{~b}$ & $41.38 \mathrm{c}$ & $27.85 \mathrm{a}$ \\
\hline Foxtail millet & $22.90 \mathrm{a}$ & $35.67 \mathrm{c}$ & $43.56 \mathrm{~d}$ & $27.30 \mathrm{~b}$ \\
\hline Proso millet & $30.97 \mathrm{a}$ & $35.80 \mathrm{ab}$ & $38.41 \mathrm{c}$ & $31.50 \mathrm{ab}$ \\
\hline Kodo millet & $24.35 \mathrm{a}$ & $28.70 \mathrm{a}$ & $38.12 \mathrm{~b}$ & $26.09 \mathrm{a}$ \\
\hline Little millet & $27.67 \mathrm{a}$ & $31.37 \mathrm{~b}$ & 39.74 & $31.36 \mathrm{~b}$ \\
\hline TranspirationRate & & & & \\
\hline Barnyard millet & $5.25 \mathrm{a}$ & $11.40 \mathrm{~b}$ & $12.77 \mathrm{c}$ & $11.61 \mathrm{~b}$ \\
\hline Foxtail millet & $5.00 \mathrm{a}$ & $7.20 \mathrm{~b}$ & $13.07 \mathrm{~d}$ & $11.22 \mathrm{c}$ \\
\hline Proso millet & $6.40 \mathrm{a}$ & $9.60 \mathrm{~b}$ & $14.74 \mathrm{~d}$ & $10.71 \mathrm{c}$ \\
\hline Kodo millet & $5.10 \mathrm{a}$ & $8.00 \mathrm{~b}$ & $13.02 \mathrm{~d}$ & $10.88 \mathrm{c}$ \\
\hline Little millet & $5.40 \mathrm{a}$ & $8.13 \mathrm{~b}$ & $13.74 \mathrm{~d}$ & $12.02 \mathrm{c}$ \\
\hline Stomatal conductance & & & & \\
\hline Barnyard millet & $0.32 \mathrm{a}$ & $1.42 \mathrm{~d}$ & $0.49 \mathrm{~b}$ & $0.79 \mathrm{c}$ \\
\hline Foxtail millet & $0.24 \mathrm{a}$ & $1.09 \mathrm{~d}$ & $0.65 \mathrm{~b}$ & $0.80 \mathrm{c}$ \\
\hline Proso millet & $0.32 \mathrm{a}$ & $1.36 \mathrm{~d}$ & $1.11 \mathrm{~b}$ & $1.23 \mathrm{c}$ \\
\hline Kodo millet & $0.26 \mathrm{a}$ & $1.13 \mathrm{~d}$ & $0.58 \mathrm{~b}$ & $0.77 \mathrm{c}$ \\
\hline Little millet & $0.27 \mathrm{a}$ & $1.12 \mathrm{~d}$ & $0.82 \mathrm{~b}$ & $1.03 \mathrm{c}$ \\
\hline
\end{tabular}

\#Growth Stages: Mean values of a trait at different growth stages were compared using Neman and Kuel's test (Newman 1939; Keuls1952). The means followed by different letter for a given trait and crop at different stages indicating significant difference at $5 \%$ probability. 
Table.2 Mean performance of small millets for different physiological traits at different growth stages

\begin{tabular}{|c|c|c|c|c|c|}
\hline \multirow[t]{2}{*}{ Crop and trait } & \multicolumn{5}{|c|}{ Crop Stages\# } \\
\hline & Vegetative & Flowering & $\begin{array}{c}\text { Grain } \\
\text { development }\end{array}$ & $\begin{array}{c}\text { Grain } \\
\text { maturation }\end{array}$ & Harvest \\
\hline \multicolumn{6}{|l|}{ Leaf number } \\
\hline Barnyard millet & $3.60 \mathrm{a}$ & $7.60 \mathrm{~b}$ & $8.20 b$ & $7.85 b$ & $6.45 b$ \\
\hline Foxtail millet & $4.80 \mathrm{a}$ & $5.8 \mathrm{bc}$ & $6.4 \mathrm{c}$ & $5.9 \mathrm{abc}$ & $5.1 \mathrm{ab}$ \\
\hline Proso millet & $6.00 \mathrm{ab}$ & $6.93 \mathrm{ab}$ & $7.63 b$ & $6.53 \mathrm{ab}$ & $5.5 \mathrm{a}$ \\
\hline Kodo millet & $4.52 \mathrm{a}$ & $4.85 \mathrm{a}$ & $5.60 \mathrm{a}$ & $5.30 \mathrm{a}$ & $4.80 \mathrm{a}$ \\
\hline Little millet & $4.80 \mathrm{a}$ & $7.40 \mathrm{~b}$ & $13.20 \mathrm{c}$ & $11.93 \mathrm{c}$ & $6.90 \mathrm{~b}$ \\
\hline \multicolumn{6}{|l|}{ Leaf Area } \\
\hline Barnyard millet & $540 \mathrm{a}$ & $673 b$ & $819 c$ & $767 b c$ & $653 b$ \\
\hline Foxtail millet & $335 \mathrm{a}$ & $520 \mathrm{~b}$ & $756 d$ & $652 c$ & $587 \mathrm{bc}$ \\
\hline Proso millet & $342 \mathrm{a}$ & $571 b$ & $790 b$ & $715 b$ & $581 b$ \\
\hline Kodo millet & $252 \mathrm{a}$ & $535 b$ & $818 c$ & $705 b c$ & $598 b$ \\
\hline Little millet & $334 a$ & $423 a b$ & $861 d$ & $689 c$ & $547 \mathrm{bc}$ \\
\hline \multicolumn{6}{|l|}{ Leaf area Index (LAI) } \\
\hline Barnyard millet & $2.41 \mathrm{a}$ & $2.99 \mathrm{ab}$ & $3.42 b c$ & $3.64 c$ & $2.91 b$ \\
\hline Foxtail millet & $1.49 \mathrm{a}$ & $2.31 b$ & $2.90 \mathrm{c}$ & $3.36 \mathrm{~d}$ & $2.61 b c$ \\
\hline Proso millet & $1.52 \mathrm{a}$ & $2.54 b$ & $3.18 b$ & $3.52 b$ & $2.58 b$ \\
\hline Kodo millet & $1.13 \mathrm{a}$ & $2.38 b$ & $3.13 b c$ & $3.64 \mathrm{c}$ & $2.655 \mathrm{v}$ \\
\hline Little millet & $1.48 \mathrm{a}$ & $1.88 \mathrm{ab}$ & $3.06 \mathrm{c}$ & $3.83 d$ & $2.43 b c$ \\
\hline \multicolumn{6}{|c|}{ Leaf area duration (LAD) } \\
\hline Barnyard millet & $27.00 \mathrm{a}$ & $32.05 \mathrm{ab}$ & $32.73 \mathrm{ab}$ & $35.28 b$ & $31.76 \mathrm{~b}$ \\
\hline Foxtail millet & $19.00 \mathrm{a}$ & $26.03 b$ & $31.28 \mathrm{c}$ & $29.83 b c$ & $26.54 b$ \\
\hline Proso millet & $20.28 \mathrm{a}$ & $28.58 \mathrm{ab}$ & $28.58 \mathrm{ab}$ & $33.47 b$ & $30.48 \mathrm{ab}$ \\
\hline Kodo millet & $17.52 \mathrm{a}$ & $27.55 b$ & $33.83 b$ & $31.45 b$ & $27.59 \mathrm{~b}$ \\
\hline
\end{tabular}




\begin{tabular}{|l|c|c|c|c|c|}
\hline Little millet & $16.82 \mathrm{a}$ & $24.70 \mathrm{~b}$ & $34.43 \mathrm{~b}$ & $31.30 \mathrm{~b}$ & $26.81 \mathrm{~b}$ \\
\hline Specific leaf weight (SLW) & & & & \\
\hline Barnyard millet & $7.22 \mathrm{a}$ & $7.65 \mathrm{ab}$ & $22.48 \mathrm{~d}$ & $16.31 \mathrm{c}$ & $11.76 \mathrm{a}$ \\
\hline Foxtail millet & $7.25 \mathrm{a}$ & $8.19 \mathrm{a}$ & $25.28 \mathrm{~d}$ & $18.59 \mathrm{c}$ & $13.33 \mathrm{~b}$ \\
\hline Proso millet & $6.98 \mathrm{a}$ & $8.10 \mathrm{a}$ & $24.70 \mathrm{~d}$ & $17.52 \mathrm{c}$ & $12.65 \mathrm{~b}$ \\
\hline Kodo millet & $6.27 \mathrm{a}$ & $7.45 \mathrm{a}$ & $23.87 \mathrm{c}$ & $16.07 \mathrm{~b}$ & $11.73 \mathrm{ab}$ \\
\hline Little millet & $7.14 \mathrm{a}$ & $7.61 \mathrm{a}$ & $22.79 \mathrm{~d}$ & $16.94 \mathrm{c}$ & $7.61 \mathrm{a}$ \\
\hline Chlorophyll 'a & & & & & \\
\hline Barnyard millet & $1.47 \mathrm{ab}$ & $1.54 \mathrm{ab}$ & $1.73 \mathrm{~b}$ & $1.34 \mathrm{a}$ & $1.26 \mathrm{a}$ \\
\hline Foxtail millet & $1.45 \mathrm{~b}$ & $1.60 \mathrm{c}$ & $1.75 \mathrm{c}$ & $1.33 \mathrm{ab}$ & $1.23 \mathrm{a}$ \\
\hline Proso millet & $1.357 \mathrm{a}$ & $1.60 \mathrm{~b}$ & $1.78 \mathrm{c}$ & $1.37 \mathrm{a}$ & $1.233 \mathrm{a}$ \\
\hline Kodo millet & $1.43 \mathrm{~b}$ & $1.62 \mathrm{~b}$ & $1.72 \mathrm{c}$ & $1.25 \mathrm{a}$ & $1.19 \mathrm{a}$ \\
\hline Little millet & $1.41 \mathrm{ab}$ & $1.51 \mathrm{bc}$ & $1.60 \mathrm{c}$ & $1.29 \mathrm{a}$ & $1.28 \mathrm{a}$ \\
\hline Chlorophyll 'b' & & & & \\
\hline Barnyard millet & $0.35 \mathrm{ab}$ & $0.42 \mathrm{ab}$ & $0.56 \mathrm{~b}$ & $0.46 \mathrm{ab}$ & $0.29 \mathrm{a}$ \\
\hline Foxtail millet & $0.40 \mathrm{a}$ & $0.44 \mathrm{a}$ & $0.78 \mathrm{a}$ & $0.61 \mathrm{a}$ & $0.30 \mathrm{a}$ \\
\hline Proso millet & $0.18 \mathrm{a}$ & $0.42 \mathrm{a}$ & $0.52 \mathrm{a}$ & $0.38 \mathrm{a}$ & $0.18 \mathrm{a}$ \\
\hline Kodo millet & $0.31 \mathrm{a}$ & $0.42 \mathrm{a}$ & $0.54 \mathrm{a}$ & $0.49 \mathrm{a}$ & $0.39 \mathrm{a}$ \\
\hline Little millet & $0.25 \mathrm{a}$ & $0.36 \mathrm{a}$ & $0.36 \mathrm{a}$ & $0.50 \mathrm{a}$ & $0.30 \mathrm{a}$ \\
\hline Total Chlorophyll & & & & & \\
\hline Barnyard millet & $1.66 \mathrm{ab}$ & $1.78 \mathrm{ab}$ & $1.92 \mathrm{~b}$ & $1.80 \mathrm{ab}$ & $1.55 \mathrm{a}$ \\
\hline Foxtail millet & $1.67 \mathrm{ab}$ & $1.77 \mathrm{bc}$ & $1.93 \mathrm{c}$ & $1.74 \mathrm{bc}$ & $1.53 \mathrm{a}$ \\
\hline Proso millet & $1.53 \mathrm{ab}$ & $1.80 \mathrm{~cd}$ & $1.91 \mathrm{~d}$ & $1.7 \mathrm{bc}$ & $1.4 \mathrm{a}$ \\
\hline Kodo millet & $1.70 \mathrm{a}$ & $1.83 \mathrm{a}$ & $2.03 \mathrm{a}$ & $1.72 \mathrm{a}$ & $1.58 \mathrm{a}$ \\
\hline Little millet & $0.58 \mathrm{~b}$ & $0.72 \mathrm{~d}$ & $0.77 \mathrm{e}$ & $0.68 \mathrm{c}$ & $0.55 \mathrm{a}$ \\
\hline Chlorophyll fluorescence $(\mathrm{Fv} / \mathrm{Fm}$ & $1.82 \mathrm{~b}$ & $1.92 \mathrm{~b}$ & $1.63 \mathrm{ab}$ & $1.48 \mathrm{a}$ \\
\hline Barnyard millet & $\mathrm{atio})$ & & & & \\
\hline & & & & & \\
\hline
\end{tabular}




\begin{tabular}{|l|c|c|c|c|c|}
\hline Foxtail millet & $0.59 \mathrm{~b}$ & $0.71 \mathrm{~d}$ & $0.77 \mathrm{e}$ & $0.69 \mathrm{c}$ & $0.57 \mathrm{a}$ \\
\hline Proso millet & $0.55 \mathrm{a}$ & $0.69 \mathrm{~b}$ & $0.75 \mathrm{c}$ & $0.67 \mathrm{~b}$ & $0.54 \mathrm{a}$ \\
\hline Kodo millet & $0.58 \mathrm{~b}$ & $0.68 \mathrm{~d}$ & $0.75 \mathrm{e}$ & $0.67 \mathrm{c}$ & $0.55 \mathrm{a}$ \\
\hline Little millet & $0.58 \mathrm{~b}$ & $0.72 \mathrm{~d}$ & $0.76 \mathrm{e}$ & $0.68 \mathrm{c}$ & $0.56 \mathrm{a}$ \\
\hline SPAD & \multicolumn{5}{|l}{} \\
\hline Barnyard millet & $37.20 \mathrm{~b}$ & $43.60 \mathrm{c}$ & $52.70 \mathrm{~d}$ & $35.50 \mathrm{~b}$ & $25.65 \mathrm{a}$ \\
\hline Foxtail millet & $38.70 \mathrm{~b}$ & $46.93 \mathrm{c}$ & $54.53 \mathrm{~d}$ & $38.47 \mathrm{~b}$ & $23.07 \mathrm{a}$ \\
\hline Proso millet & $34.07 \mathrm{~b}$ & $43.47 \mathrm{c}$ & $51.87 \mathrm{~d}$ & $33.70 \mathrm{~b}$ & $16.30 \mathrm{a}$ \\
\hline Kodo millet & $35.20 \mathrm{~b}$ & $42.45 \mathrm{bc}$ & $49.60 \mathrm{c}$ & $33.60 \mathrm{~b}$ & $22.65 \mathrm{a}$ \\
\hline Little millet & $35.47 \mathrm{~b}$ & $44.77 \mathrm{c}$ & $52.77 \mathrm{~d}$ & $35.03 \mathrm{~b}$ & $25.43 \mathrm{a}$ \\
\hline Root length $(\mathrm{cm})$ & & & & & \\
\hline Barnyard millet & $6.00 \mathrm{a}$ & $17.10 \mathrm{~b}$ & $17.60 \mathrm{~b}$ & $17.95 \mathrm{~b}$ & $18.05 \mathrm{~b}$ \\
\hline Foxtail millet & $5.73 \mathrm{a}$ & $9.87 \mathrm{~b}$ & $15.80 \mathrm{c}$ & $16.50 \mathrm{c}$ & $16.60 \mathrm{c}$ \\
\hline Proso millet & $6.67 \mathrm{a}$ & $14.23 \mathrm{~b}$ & $16.10 \mathrm{c}$ & $16.27 \mathrm{c}$ & $16.43 \mathrm{c}$ \\
\hline Kodo millet & $5.75 \mathrm{a}$ & $11.65 \mathrm{~b}$ & $14.70 \mathrm{c}$ & $15.35 \mathrm{c}$ & $16.00 \mathrm{c}$ \\
\hline Little millet & $5.55 \mathrm{a}$ & $12.40 \mathrm{~b}$ & $15.40 \mathrm{c}$ & $15.50 \mathrm{c}$ & $15.80 \mathrm{c}$ \\
\hline
\end{tabular}

Growth Stages: Mean values of a trait at different growth stages were compared using Neman and Kuel's test (Newman 1939; Keuls1952). The means followed by different letter for a given trait and crop at different stages indicating significant difference at $5 \%$ probability. 
Table.3 Physiological traits - Number of leaves, Leaf area $\left(\mathrm{cm}^{2}\right)$ and Leaf area index of small millets at different growth stages

\begin{tabular}{|c|c|c|c|c|c|c|c|c|c|c|c|c|c|c|c|}
\hline \multirow[t]{2}{*}{ Crop } & \multicolumn{5}{|c|}{ Number of leaves } & \multicolumn{5}{|c|}{ Leaf area $\left(\mathrm{cm}^{2}\right)$} & \multicolumn{5}{|c|}{ Leaf area index } \\
\hline & I & II & III & IV & $\mathbf{V}$ & I & II & III & IV & $\mathbf{V}$ & I & II & III & IV & $\mathbf{V}$ \\
\hline \multicolumn{16}{|c|}{ Banyard millet } \\
\hline $\mathrm{CO} 1$ & 3.4 & 7.2 & 7.6 & 7.0 & 6.3 & 510.43 & 656.29 & 790.66 & 744.21 & 614.54 & 1.35 & 2.17 & 2.54 & 3.04 & 2.46 \\
\hline $\mathrm{CO} 2$ & 3.8 & 8.0 & 8.8 & 8.7 & 6.6 & 570.88 & 689.77 & 849.18 & 791.49 & 691.88 & 1.43 & 2.63 & 3.10 & 3.37 & 2.60 \\
\hline \multicolumn{16}{|c|}{ Foxtail millet } \\
\hline CO 5 & 4.4 & 5.4 & 6.3 & 6.0 & 4.8 & 305.12 & 470.96 & 729.44 & 610.73 & 588.15 & 1.77 & 2.82 & 3.89 & 4.14 & 2.68 \\
\hline $\mathrm{CO} 6$ & 4.7 & 5.6 & 6.0 & 5.9 & 5.0 & 330.15 & 499.61 & 740.61 & 651.83 & 579.38 & 1.36 & 2.09 & 2.71 & 3.24 & 2.61 \\
\hline $\mathrm{CO} 7$ & 5.4 & 6.6 & 6.8 & 5.8 & 5.6 & 370.65 & 588.11 & 797.14 & 694.32 & 594.88 & 1.47 & 2.22 & 2.90 & 3.29 & 2.58 \\
\hline \multicolumn{16}{|c|}{ Proso millet } \\
\hline $\mathrm{CO} 3$ & 5.7 & 6.6 & 7.3 & 5.3 & 5.1 & 304.24 & 489.33 & 683.44 & 570.82 & 554.12 & 1.65 & 2.61 & 3.09 & 3.54 & 2.64 \\
\hline $\mathrm{CO} 4$ & 5.9 & 6.8 & 7.1 & 6.6 & 5.3 & 322.11 & 591.02 & 757.61 & 697.25 & 585.65 & 1.25 & 2.58 & 3.26 & 3.94 & 2.69 \\
\hline CO 5 & 6.5 & 7.4 & 8.5 & 7.7 & 6.1 & 399.34 & 633.91 & 930.57 & 875.96 & 603.59 & 1.00 & 2.18 & 3.00 & 3.33 & 2.62 \\
\hline \multicolumn{16}{|c|}{ Kodo millet } \\
\hline $\mathrm{CO} 3$ & 4.6 & 5.5 & 6.6 & 6.2 & 5.4 & 280.19 & 580.51 & 887.11 & 733.77 & 606.29 & 1.49 & 1.69 & 2.63 & 3.48 & 2.20 \\
\hline APK & 3.9 & 4.2 & 4.6 & 4.4 & 4.2 & 225.33 & 489.77 & 749.82 & 675.48 & 590.19 & 1.40 & 1.78 & 2.84 & 3.56 & 2.31 \\
\hline \multicolumn{16}{|c|}{ Little millet } \\
\hline $\mathrm{CO} 2$ & 4.1 & 7.1 & 12.0 & 10.5 & 6.1 & 335.61 & 380.14 & 783.59 & 590.77 & 494.11 & 1.56 & 2.17 & 3.71 & 4.44 & 2.79 \\
\hline $\mathrm{CO} 3$ & 3.7 & 7.4 & 13.2 & 12.3 & 6.9 & 315.19 & 399.46 & 800.11 & 640.08 & 519.36 & 2.27 & 2.92 & 3.31 & 3.51 & 2.73 \\
\hline $\mathrm{CO} 4$ & 6.6 & 7.7 & 14.4 & 13.0 & 7.7 & 350.15 & 488.27 & 999.87 & 835.14 & 627.37 & 2.54 & 3.07 & 3.52 & 3.77 & 3.08 \\
\hline Mean & 4.8 & 6.6 & 8.4 & 7.6 & 5.8 & 355.33 & 535.17 & 807.62 & 700.91 & 588.42 & 1.58 & 2.38 & 3.12 & 3.59 & 2.61 \\
\hline SED & 0.017 & 0.018 & 0.048 & 0.044 & 0.015 & 1.521 & 1.567 & 1.437 & 1.518 & 0.794 & 0.006 & 0.007 & 0.006 & 0.006 & 0.003 \\
\hline $\mathrm{CD}(0.05)$ & 0.036 & 0.037 & 0.100 & 0.091 & 0.032 & 3.139 & 3.234 & 2.967 & 3.134 & 1.640 & 1.35 & 2.17 & 2.54 & 3.04 & 2.46 \\
\hline
\end{tabular}


Table.4 Physiological traits - Leaf area duration (days), Specific Leaf Weight $\left(\mathrm{mg} / \mathrm{cm}^{-2}\right)$ and Root length ( $\left.\mathrm{cm}\right)$ of small millets at different growth stages

\begin{tabular}{|c|c|c|c|c|c|c|c|c|c|c|c|c|c|c|}
\hline \multirow[t]{2}{*}{ Crop } & \multicolumn{4}{|c|}{ Leaf area duration (days) } & \multicolumn{5}{|c|}{ Specific Leaf Weight $\left(\mathrm{mg} / \mathrm{cm}^{-2}\right)$} & \multicolumn{5}{|c|}{ Root length $(\mathrm{cm})$} \\
\hline & I & II & III & IV & I & II & III & IV & $\mathbf{V}$ & I & II & III & IV & V \\
\hline \multicolumn{15}{|c|}{ Banyard millet } \\
\hline $\mathrm{CO} 2$ & 20.30 & 28.65 & 32.35 & 29.85 & 6.6 & 7.7 & 14.4 & 13.0 & 7.7 & 6.5 & 17.7 & 18.5 & 18.6 & 18.7 \\
\hline \multicolumn{15}{|c|}{ Foxtail millet } \\
\hline $\mathrm{CO} 7$ & 18.45 & 25.60 & 30.95 & 29.35 & 5.7 & 6.6 & 7.3 & 5.3 & 5.1 & 6.5 & 13.5 & 16.3 & 16.5 & 16.6 \\
\hline \multicolumn{15}{|l|}{ Proso millet } \\
\hline $\mathrm{CO} 3$ & 21.3 & 28.50 & 33.15 & 30.90 & 3.4 & 7.2 & 7.6 & 7.0 & 6.3 & 5.9 & 13.2 & 15.5 & 15.7 & 15.9 \\
\hline $\mathrm{CO} 4$ & 19.15 & 29.20 & 36.00 & 33.15 & 3.8 & 8.0 & 8.8 & 8.7 & 6.6 & 6.5 & 13.9 & 16.3 & 16.4 & 16.6 \\
\hline $\mathrm{CO} 5$ & 15.9 & 25.90 & 31.65 & 29.75 & 4.4 & 5.4 & 6.3 & 6.0 & 4.8 & 7.6 & 15.6 & 16.5 & 16.7 & 16.8 \\
\hline \multicolumn{15}{|l|}{ Little millet } \\
\hline $\mathrm{CO} 2$ & 18.65 & 29.40 & 40.75 & 36.15 & 4.6 & 5.5 & 6.6 & 6.2 & 5.4 & 5.6 & 11.5 & 12.2 & 15.5 & 15.8 \\
\hline $\mathrm{CO} 3$ & 25.95 & 31.15 & 34.10 & 31.20 & 3.9 & 4.2 & 4.6 & 4.4 & 4.2 & 5.4 & 12.3 & 15.3 & 15.4 & 15.8 \\
\hline $\mathrm{CO} 4$ & 28.05 & 32.95 & 36.45 & 34.25 & 4.1 & 7.1 & 12.0 & 10.5 & 6.1 & 5.7 & 12.5 & 15.5 & 15.6 & 15.8 \\
\hline Mean & 19.79 & 27.47 & 33.52 & 31.02 & 4.8 & 6.6 & 8.4 & 7.6 & 5.8 & 355.33 & 535.17 & 807.62 & 700.91 & 588.42 \\
\hline SED & 0.063 & 0.062 & 0.063 & 0.042 & 0.017 & 0.018 & 0.048 & 0.044 & 0.015 & 1.521 & 1.567 & 1.437 & 1.518 & 0.794 \\
\hline $\mathrm{CD}(0.05)$ & 0.130 & 0.129 & 0.131 & 0.088 & 0.036 & 0.037 & 0.100 & 0.091 & 0.032 & 3.139 & 3.234 & 2.967 & 3.134 & 1.640 \\
\hline
\end{tabular}


Table.5 Physiological traits - Chlorophyll ' $a$ ' ( $\left.\mathrm{mg} \mathrm{g}^{-1}\right)$, Chlorophyll 'b' ( $\left.\mathrm{mg} \mathrm{g}^{-1}\right)$ and Total Chlorophyll ( $\left.\mathrm{mg} \mathrm{g}^{-1}\right)$ content of small millets at different growth stages

\begin{tabular}{|c|c|c|c|c|c|c|c|c|c|c|c|c|c|c|c|}
\hline \multirow[t]{2}{*}{ Crop } & \multicolumn{5}{|c|}{ Chlorophyll 'a' $\left(\mathrm{mg} \mathrm{g}^{-1}\right)$} & \multicolumn{5}{|c|}{ Chlorophyll 'b' ( $\left.\mathrm{mg} \mathrm{g}^{-1}\right)$} & \multicolumn{5}{|c|}{ Total Chlorophyll $\left(\mathrm{mg} \mathrm{g}^{-1}\right)$} \\
\hline & $\mathbf{I}$ & II & III & IV & $\mathbf{V}$ & I & II & III & IV & $\mathbf{V}$ & I & II & III & IV & $\mathbf{V}$ \\
\hline \multicolumn{16}{|c|}{ Banyard millet } \\
\hline $\mathrm{CO} 1$ & 1.43 & 1.47 & 1.62 & 1.32 & 1.20 & 0.31 & 0.36 & 0.50 & 0.42 & 0.28 & 1.63 & 1.73 & 1.88 & 1.74 & 1.48 \\
\hline $\mathrm{CO} 2$ & 1.51 & 1.60 & 1.83 & 1.37 & 1.32 & 0.39 & 0.48 & 0.61 & 0.49 & 0.30 & 1.69 & 1.82 & 1.95 & 1.86 & 1.62 \\
\hline \multicolumn{16}{|c|}{ Foxtail millet } \\
\hline CO 5 & 1.39 & 1.51 & 1.65 & 1.28 & 1.19 & 0.37 & 0.41 & 0.58 & 0.57 & 0.31 & 1.60 & 1.72 & 1.89 & 1.85 & 1.50 \\
\hline $\mathrm{CO} 6$ & 1.45 & 1.55 & 1.78 & 1.31 & 1.22 & 0.38 & 0.45 & 0.70 & 0.31 & 0.20 & 1.66 & 1.78 & 1.92 & 1.62 & 1.42 \\
\hline $\mathrm{CO} 7$ & 1.51 & 1.75 & 1.83 & 1.41 & 1.28 & 0.44 & 0.45 & 1.05 & 0.95 & 0.39 & 1.75 & 1.82 & 1.97 & 1.74 & 1.67 \\
\hline \multicolumn{16}{|c|}{ Proso millet } \\
\hline $\mathrm{CO} 3$ & 1.34 & 1.66 & 1.73 & 1.33 & 1.22 & 0.17 & 0.46 & 0.51 & 0.32 & 0.11 & 1.51 & 1.72 & 1.87 & 1.65 & 1.33 \\
\hline $\mathrm{CO} 4$ & 1.39 & 1.40 & 1.78 & 1.37 & 1.20 & 0.18 & 0.41 & 0.25 & 0.24 & 0.18 & 1.57 & 1.81 & 1.91 & 1.61 & 1.38 \\
\hline CO 5 & 1.34 & 1.73 & 1.82 & 1.41 & 1.28 & 0.17 & 0.46 & 0.51 & 0.32 & 0.11 & 1.52 & 1.88 & 1.95 & 1.72 & 1.61 \\
\hline \multicolumn{16}{|c|}{ Kodo millet } \\
\hline $\mathrm{CO} 3$ & 1.41 & 1.66 & 1.76 & 1.30 & 1.18 & 0.35 & 0.51 & 0.64 & 0.59 & 0.48 & 1.68 & 1.77 & 1.95 & 1.85 & 1.66 \\
\hline APK & 1.45 & 1.57 & 1.68 & 1.20 & 1.20 & 0.27 & 0.33 & 0.43 & 0.38 & 0.30 & 1.72 & 1.90 & 2.11 & 1.58 & 1.50 \\
\hline \multicolumn{16}{|c|}{ Little millet } \\
\hline $\mathrm{CO} 2$ & 1.31 & 1.48 & 1.56 & 1.22 & 1.21 & 0.21 & 0.30 & 0.37 & 0.16 & 0.10 & 1.52 & 1.78 & 1.93 & 1.38 & 1.31 \\
\hline $\mathrm{CO} 3$ & 1.43 & 1.50 & 1.54 & 1.29 & 1.31 & 0.25 & 0.34 & 0.60 & 0.46 & 0.17 & 1.68 & 1.84 & 1.90 & 1.75 & 1.48 \\
\hline $\mathrm{CO} 4$ & 1.49 & 1.56 & 1.70 & 1.36 & 1.31 & 0.28 & 0.45 & 0.79 & 0.88 & 0.34 & 1.74 & 1.83 & 1.94 & 1.76 & 1.65 \\
\hline Mean & 1.41 & 1.57 & 1.71 & 1.32 & 1.04 & 0.29 & 0.41 & 0.60 & 0.49 & 0.27 & 1.64 & 1.80 & 1.94 & 1.70 & 1.51 \\
\hline SED & 0.0011 & 0.0017 & 0.0016 & 0.0011 & 0.0008 & 0.0015 & 0.0010 & 0.0034 & 0.0038 & 0.0018 & 0.0014 & 0.0009 & 0.0010 & 0.0022 & 0.0021 \\
\hline $\mathrm{CD}(0.05)$ & 0.0022 & 0.0035 & 0.0033 & 0.0022 & 0.0017 & 0.0030 & 0.0021 & 0.0071 & 0.0078 & 0.0078 & 0.0029 & 0.0019 & 0.0020 & 0.0045 & 0.0042 \\
\hline
\end{tabular}


Table.6Chlorophyll fluorescence (Fv/Fm ratio), SPAD values, Yield potential and harvest index of small millets

\begin{tabular}{|c|c|c|c|c|c|c|c|c|c|c|c|c|c|c|c|}
\hline \multirow[t]{2}{*}{ Crop } & \multicolumn{5}{|c|}{ Chlorophyll fluorescence (Fv/Fm ratio) } & \multicolumn{5}{|c|}{ SPAD values } & \multicolumn{5}{|c|}{ Yield potential and Harvest index } \\
\hline & I & II & III & IV & $\mathbf{V}$ & I & II & III & IV & $\mathbf{V}$ & $\begin{array}{c}\text { Days to } \\
50 \% \\
\text { flowering }\end{array}$ & $\begin{array}{l}\text { Plant } \\
\text { height }\end{array}$ & $\begin{array}{c}\text { Days to } \\
\text { maturity } \\
\text { (days) }\end{array}$ & $\begin{array}{l}\text { Grain } \\
\text { yield } \\
\text { (Kg/ha) }\end{array}$ & $\begin{array}{l}\text { Harvest } \\
\text { index }\end{array}$ \\
\hline \multicolumn{16}{|c|}{ Banyard millet } \\
\hline $\mathrm{CO} 1$ & 0.581 & 0.723 & 0.774 & 0.679 & 0.552 & 36.2 & 41.5 & 50.6 & 34.7 & 24.8 & 52 & 113 & 95 & 2197 & 0.35 \\
\hline $\mathrm{CO} 2$ & 0.583 & 0.725 & 0.778 & 0.682 & 0.555 & 38.2 & 45.7 & 54.8 & 36.3 & 26.5 & 48 & 120 & 92 & 3091 & 0.38 \\
\hline \multicolumn{16}{|c|}{ Foxtail millet } \\
\hline CO 5 & 0.586 & 0.708 & 0.770 & 0.692 & 0.569 & 35.2 & 45.1 & 51.1 & 38.8 & 22.1 & 43 & 106 & 89 & 2716 & 0.27 \\
\hline $\mathrm{CO} 6$ & 0.589 & 0.715 & 0.772 & 0.695 & 0.572 & 38.4 & 47.2 & 54.0 & 37.5 & 20.4 & 43 & 106 & 87 & 2883 & 0.28 \\
\hline $\mathrm{CO} 7$ & 0.592 & 0.714 & 0.775 & 0.697 & 0.574 & 42.5 & 48.5 & 58.5 & 39.1 & 26.7 & 40 & 111 & 83 & 3499 & 0.31 \\
\hline \multicolumn{16}{|c|}{ Proso millet } \\
\hline $\mathrm{CO} 3$ & 0.526 & 0.663 & 0.731 & 0.675 & 0.521 & 32.8 & 44.2 & 49.6 & 30.4 & 17.2 & 47 & 105 & 94 & 2883 & 0.39 \\
\hline $\mathrm{CO} 4$ & 0.562 & 0.669 & 0.748 & 0.664 & 0.537 & 33.1 & 40.6 & 51.3 & 34.5 & 16.4 & 45 & 106 & 93 & 2666 & 0.39 \\
\hline $\mathrm{CO} 5$ & 0.567 & 0.674 & 0.769 & 0.673 & 0.558 & 36.3 & 45.6 & 54.7 & 36.2 & 15.3 & 44 & 113 & 90 & 3083 & 0.4 \\
\hline \multicolumn{16}{|c|}{ Kodo millet } \\
\hline $\mathrm{CO} 3$ & 0.575 & 0.681 & 0.752 & 0.674 & 0.543 & 37.4 & 46.4 & 52.5 & 35.9 & 22.1 & 60 & 96 & 115 & 2016 & 0.41 \\
\hline APK & 0.577 & 0.683 & 0.755 & 0.677 & 0.546 & 33.0 & 38.5 & 46.7 & 31.3 & 23.2 & 65 & 94 & 122 & 1133 & 0.39 \\
\hline \multicolumn{16}{|c|}{ Little millet } \\
\hline $\mathrm{CO} 2$ & 0.575 & 0.720 & 0.760 & 0.675 & 0.558 & 34.2 & 42.2 & 50.7 & 33.5 & 24.7 & 48 & 103 & 86 & 2466 & 0.35 \\
\hline $\mathrm{CO} 3$ & 0.578 & 0.723 & 0.764 & 0.679 & 0.560 & 35.1 & 44.5 & 52.4 & 36.2 & 25.4 & 47 & 105 & 83 & 2499 & 0.36 \\
\hline $\mathrm{CO} 4$ & 0.581 & 0.726 & 0.769 & 0.682 & 0.561 & 37.1 & 47.6 & 55.2 & 35.4 & 26.2 & 43 & 106 & 80 & 2774 & 0.38 \\
\hline Mean & 0.574 & 0.701 & 0.763 & 0.680 & 0.554 & 36.11 & 44.43 & 52.47 & 35.37 & 22.38 & 48 & 107 & 93 & 2608 & 0.36 \\
\hline SED & 0.0003 & 0.0004 & 0.0002 & 0.0002 & 0.0002 & 0.044 & 0.048 & 0.049 & 0.041 & 0.064 & 0.117 & 0.113 & 0.201 & 9.630 & 0.001 \\
\hline $\mathrm{CD}(0.05)$ & 0.0006 & 0.0008 & 0.0004 & 0.0004 & 0.0005 & 0.091 & 0.099 & 0.101 & 0.086 & 0.113 & 0.241 & 0.234 & 0.413 & 19.87 & 0.002 \\
\hline
\end{tabular}


Table.7 Gas exchange parameters of small millets at different growth stages

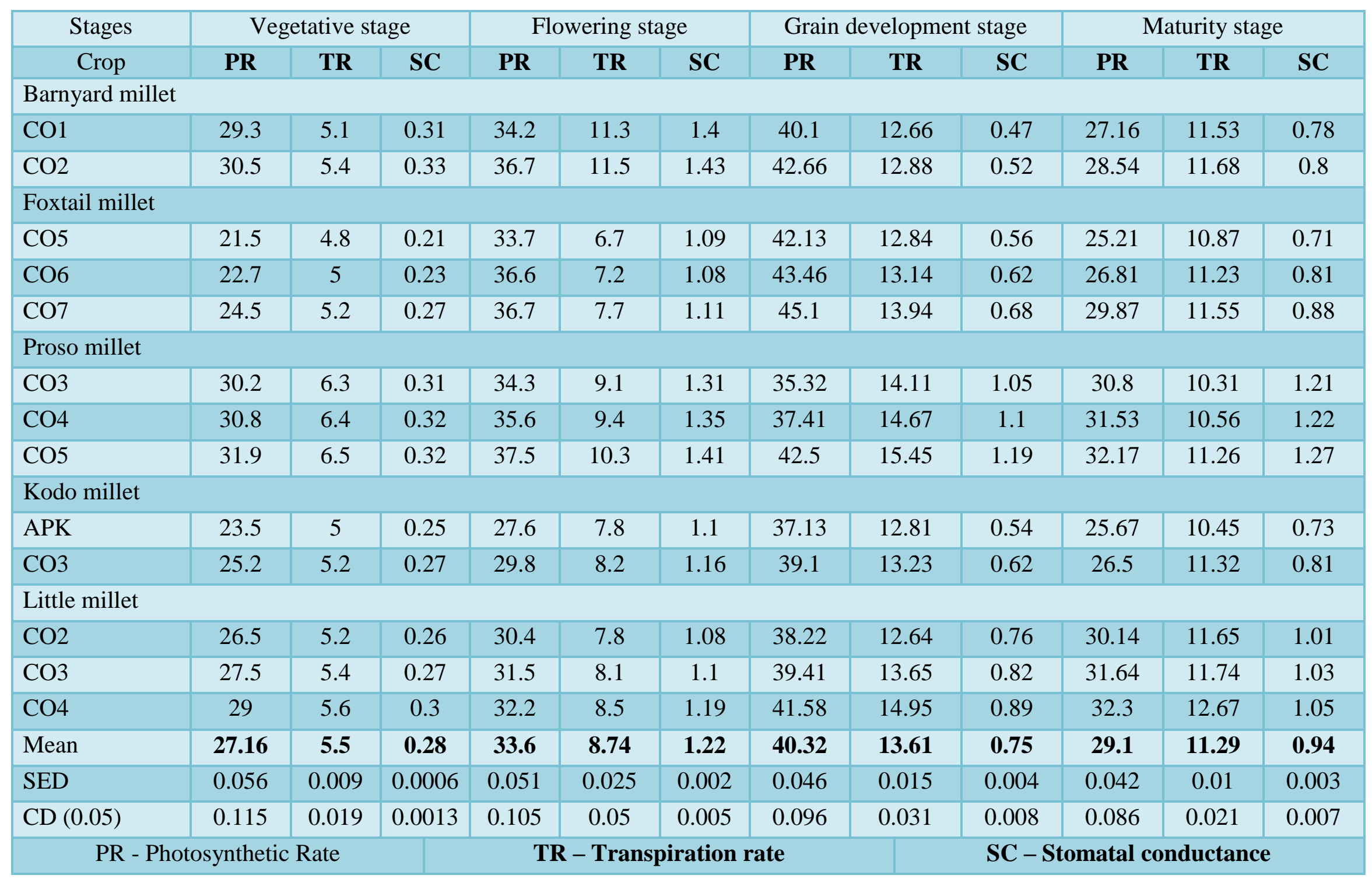


Small millets are climate resilient crops, and are less affected by insect pests and diseases and abiotic stress. However, small millets cultivation and consumption has been declined mainly due to limited productivity, high drudgery involved in their processing and negative perceptions of small millets as a food for the poor. In this study, we assessed the physiological traits at different growth stages and their relationship with grain yield. Understanding the physiological traits in crops plants at different stages helps to understand their adaptations and crop characteristics. Leaf area is the fundamental determinant of the rate of photosynthesis of any plant and the optimum leaf area development aids in effective interception of light energy and facilitates higher dry matter production.

The nature of the foliage cover is an important factor in determining the efficiency with which the available solar radiation is used in primary production (Loomis and Williams, 1969). The leaf area index is an important measure of canopy structure because crop morphology, leaf orientation and distribution influence LAI. Leaf area duration is the integral of leaf area index over time. Formation of optimum photosynthetic area and maintenance of photosynthetically active leaves for a longer duration, especially during the reproductive phase of crop, are essential for increasing the photosynthetic rate, dry matter accumulation and grain yield (Watson, 1958). Specific leaf weight plays an important role in leaf and plant functioning and is related to species strategies of resource acquisition and use. Specific leaf weight, a measure of leaf thickness, has been reported to have a strong positive correlation with leaf photosynthesis of several crops as reported by Bowes et al. (1972).

Photosynthetic pigments are composed of chlorophylls a, b and total and the main functions are interception and storage of light energy by inductive resonance through antenna complexes and consequent electron transport carried out by the Photosystem II (Taiz and Zeiger, 2002). The efficiency of leaves to produce assimilates and its persistence depends largely on photosynthetic pigments, of which, the leaf chlorophyll content is of the prime importance, which is directly associated with the increase in PSII photochemistry, photosynthate production and dry matter accumulation. Hence, measurement of chlorophylls indirectly explains the efficiency of the photosynthesis and photosynthates production. The chloroplast in green plants constitutes the photosynthetic apparatus. Chlorophylls and other photosynthetic pigments are found in the form of protein pigment complexes mainly in thylakoid membranes of grana. Photosynthetic pigments play major role in plant productivity, as they are responsible for capturing light energy and using it as a driving force for producing the assimilates. Chlorophyll index permits a rapid and non-destructive determination of leaf chlorophyll content by measuring leaf transmittance using SPAD meter.

Reduction in transpiration rate under water deficit conditions leads to reduce the photosynthetic rate by inhibition of $\mathrm{CO}_{2}$ entry into the chloroplast through the stomata. The chlorophyll fluorescence is an important measurement of photosynthetic efficiency of crops. The high Fv/ Fm ratio is proportional to quantum yield and showing high degree of photosynthesis (Gitelson et al., 1999). Fluorescence yield will be high when PS II reaction centre is least damaged by photoinhibition. In this study, Several physiological traits such as leaf number, leaf area, specific leaf weight, chlorophylls a, b, and total chlorophyll, SPAD reading, photosynthetic rate and transpiration rate have reached their maximum value at grain development stage in all the crops, while leaf area index was the maximum at grain development stage, highest root length was achieved at maturity stage, and highest stomatal conductance was at flowering stage. Among different cultivars within the each crop, a cultivar having high leaf number, leaf area, leaf area index, leaf area duration, specific leaf weight, chlorophyll a, b, and total chlorophyll, chlorophyll florescence ratio, 
SPAD reading, photosynthetic rate, transpiration rate, stomatal conductance and root length, had produced higher grain yield. This shows importance of these traits in for enhanced yields in small millets.

\section{References}

Arnon, I. 1949. Crop production in dry regions . Background and principles. Leonard Hill books. London. 650p

Bowes, G.W., L. Orgen and R.H. Hageman. 1972. Light saturated photosynthesis rate, RuBP carboxylase activity and specific leaf weight in soybeans grown under different light intensities. Crop Science.12: 77-79.

Gitleson, A.A., C. Buschmann and H.K. Lichtenthaler. 1999. Leaf chlorophyll fluorescence corrected for reabsorption by means of absorption and reflectance measurements. Journal of Plant Physiology. 152: 283-296.

Gomez, K.A. and A.A. Gomez. 2010. Statistical procedure for agricultural research. New York, Wiley Inter-Science Publications.

Keuls, M. 1952. The use of the "Studentized range" in connection with an analysis of variance. Euphytica 1:112-122.

Loomis, R. S., and W. A. Williams. 1969. Productivity and the morphology of crop stands: patterns with leaves. In Eastin, J. D., et al., eds., Physiological aspects of crop yield. American Society of Agronomy. Madison: 27-47.

Newman, D. 1939. The distribution of range in samples from a normal population expressed in terms of an independent estimate of standard deviation. Biometrika. 31:20-30.

Pearce, R.B., R.H. Brown and R.E. Blaster. 1968. Photosynthesis of alfalfa leaves as influenced by age and environment. Crop Science. 8: 677-680.

Power, J.E., W.O. Wills, D.L. Granes and G.A. Reichman. 1967. Effect of soil temperature, phosphorus and plant age on growth analysis of barley. Agronomy Journal. 59: 231-234.

Taiz, L., and Zeiger, E., (2002). Plant physiology, third ed. Sinaur, sunderland. Upadhyaya, H.D., C.L.L Gowda, V.G. Reddy and S. Singh. 2008. Diversity of small millets germplasm in genebank at ICRISAT. In: 5 th International Symposium on New Crops and Uses: their role in a rapidly changing world, 34 September, 2007, University of Southampton, Southampton, UK.

Vetriventhan, M., H.D.Upadhyaya, S.L. Dwived, S.K. Pattanashetti, and S.K. Singh. 2015. Finger and foxtail millets. p. 291-319. In Singh, M., Upadhyaya, H.D. (eds.), Genetic and Genomic Resources for Grain Cereals Improvement. Oxford: Academic Press.

Watson, D.J. 1952. The physiological basis for variation in yield. Advances in Agronomy. 101-145.

Williams, R.F. 1946. The physiology of plant growth with special referance to the concept of net assimilation rate. Annals of Botony. 10: 41-71.

\section{How to cite this article:}

Samundeswari, R D Durga Devi, P Jayakumar, Jeyapandiyan, N. Assessment of Physiological Basis Of Yield Variation In Small Millets Under Rainfed Condition Int.J.Curr.Microbiol.App.Sci. 7(07): 2453-2466. doi: https://doi.org/10.20546/ijcmas.2018.707.287 